\title{
A HIGH-ORDER APPROXIMATION METHOD FOR SEMILINEAR PARABOLIC EQUATIONS ON SPHERES
}

\author{
HOLGER WENDLAND
}

\begin{abstract}
We analyse a discretisation method for solving (systems of) semilinear parabolic equations on Euclidean spheres. The approximation method is based upon a discretisation in space using spherical basis functions and can hence be of arbitrary order in space, provided that the true solution is sufficiently smooth, which is, at least locally in time, true for semilinear parabolic problems. We rigorously prove stability and convergence of the semi-discrete approximation, if basis functions with a prescribed Sobolev regularity are employed. For discretisation in time, we give two examples and prove the expected convergence orders in space and time for the fully discretised system.
\end{abstract}

\section{INTRODUCTION}

We are interested in numerically solving (systems) of semilinear parabolic partial differential equations on the sphere $\mathbb{S}^{d-1} \subseteq \mathbb{R}^{d}$. This means we are looking for solutions $u:(0, T] \times \mathbb{S}^{d-1} \rightarrow \mathbb{R}^{n}$ of reaction diffusion equations of the form

$$
\begin{aligned}
\partial_{t} u+L u & =F(u) & & \text { on }(0, T] \times \mathbb{S}^{d-1}, \\
u(0, \cdot) & =u_{0} & & \text { on } \mathbb{S}^{d-1} .
\end{aligned}
$$

Here, $L$ denotes a second order elliptic operator. The reaction term is described by a smooth function $F: \mathbb{R}^{n} \rightarrow \mathbb{R}^{n}$ and the time interval $[0, T]$ as well as the initial data $u_{0}: \mathbb{S}^{d-1} \rightarrow \mathbb{R}^{n}$ are given. Our method is a high-order meshfree method based upon radial basis functions. Our method is in principle not restricted to the sphere but works on arbitrary compact Riemannian manifolds and also bounded Euclidean domains. However, the analysis presented in this paper will be particularly derived for the sphere as the spatial domain.

Particular examples of equations we are interested in comprise the Allen-Cahn equation with double wells potential. The Allen-Cahn equation, which has been introduced in [1, represents a diffuse interface model or phase field approach for studying the evolution of a diffuse phase boundary between two phases of, for example, crystalline solids. On the sphere, the Allen-Cahn equation takes the form

$$
\begin{aligned}
\partial_{t} u-\Delta_{*} u & =F(u)=\frac{1}{\epsilon^{2}} u\left(1-u^{2}\right) & & \text { on }(0, T] \times \mathbb{S}^{d-1}, \\
u(0, \cdot) & =u_{0} & & \text { on } \mathbb{S}^{d-1} .
\end{aligned}
$$

Here, the nonlinearity on the right-hand side is given by $F(u)=W^{\prime}(u)$ with the double well potential $W: \mathbb{R} \rightarrow \mathbb{R}$ satisfying $W^{\prime}(u)=\frac{1}{\epsilon^{2}} u\left(1-u^{2}\right)$ with a given parameter $\epsilon>0$ and $\Delta_{*}$ denotes the Laplace-Beltrami operator.

Received by the editor September 20, 2010 and, in revised form, August 15, 2011.

2010 Mathematics Subject Classification. Primary 35K58, 65M12, 65M15, 65M20 . 
Other examples, which are covered by our theory, comprise systems of semilinear parabolic equations such as activator-inhibitor models like the Schnakenberg model or the Gray-Scott model; see for example [15]:

$$
\begin{aligned}
\partial_{t} u & =D_{1} \Delta_{*} u-u v^{2}+\widetilde{\gamma}(1-u) \\
\partial_{t} v & =D_{2} \Delta_{*} v+u v^{2}-(\widetilde{\gamma}+\kappa) v \\
u(0, \cdot) & =u_{0} \\
v(0, \cdot) & =v_{0}
\end{aligned}
$$

$$
\begin{aligned}
& \text { on }(0, T] \times \mathbb{S}^{d-1}, \\
& \text { on }(0, T] \times \mathbb{S}^{d-1}, \\
& \text { on } \mathbb{S}^{d-1}, \\
& \text { on } \mathbb{S}^{d-1} \text {. }
\end{aligned}
$$

Here, $\widetilde{\gamma}$ indicates the feed rate and $\kappa$ gives the rate constant of the second reaction. These models, where the unknowns represent certain chemical concentrations, allow us to describe the interaction between two (or more) of such chemicals and are, for example, used to investigate the formation of pattern in animal coats and skins but are also used to model cancer growth.

There is a fast growing literature on solving partial differential equations using meshfree methods in general (see for example [2]) and using radial basis function or kernel-based methods in particular (see for example the literature in [4]). Amongst those, there are only a few papers, which deal with partial differential equation on spheres (see for example [6, 7, 9, 11]) though the number is steadily increasing. More recent work, including large-scale applications, is described in [5]. Most of these papers are of numerical nature, lacking a thorough mathematical analysis, or deal with time-independent problems.

This paper is organised as follows. In the rest of this section, we will introduce the necessary material for working on the sphere. Then, we will discuss material on reaction diffusion equations as required for our approximation scheme. In the third section we will derive our approximation scheme and show that it is both a collocation and a Galerkin scheme. The fourth section is devoted to a rigorous mathematical analysis of the semi-discretisation, while section 5 deals with examples of a full discretisation. Numerical examples can be found in the above mentioned papers but will also be the subject of a future paper.

Error estimates will be given in the natural norm of the reproducing kernel Hilbert space, which is a consequence of interpreting the collocation method as a Galerkin method in this norm. However, the Sobolev embedding theorem also immediately grants estimates in the maximum norm, though these are not optimal and hence require further research.

The results of this paper can also be seen in the context of improving time stepping stability and accuracy for finite difference methods based upon radial basis functions (see [8]).

1.1. Basic information on the sphere. We will study equations on the $d$-variate unit sphere given by $\mathbb{S}^{d-1}:=\left\{x \in \mathbb{R}^{d}:\|x\|_{2}=1\right\} \subseteq \mathbb{R}^{d}$. It has surface area $\omega_{d-1}=\frac{2 \pi^{d / 2}}{\Gamma(d / 2)}$. On $\mathbb{S}^{d-1}$, we will use the usual inner product

$$
\langle f, g\rangle_{L_{2}}:=\int_{\mathbb{S}^{d-1}} f(x) g(x) d S(x),
$$

where $d S(x)$ is given by the standard measure on the sphere.

The distance between two points $x, y \in \mathbb{S}^{d-1}$ is the geodesic distance, which is the length of the shorter part of the great circle joining $x$ and $y$, or in other words, $\operatorname{dist}(x, y)=\arccos \left(x^{T} y\right)$. 
The restriction of the standard Laplace operator $\Delta=\sum_{j=1}^{d} \partial_{j j}^{2}$ to the sphere gives the well-known Laplace-Beltrami operator, which can be written without a parametrisation using the standard Laplace and gradient operator and the Hessian matrix $H(u)=\left(\partial_{i j} u\right)$ :

$$
\Delta_{*} u(x)=\Delta u(x)-(d-1) x^{T} \nabla u(x)-x^{T} H(u) x .
$$

It is well known that the eigenvalues of $-\Delta_{*}$ are given as

$$
\lambda_{\ell}=\ell(\ell+d-2), \quad \ell \in \mathbb{N}_{0},
$$

and that the corresponding eigenfunctions are (spherical) polynomials called spherical harmonics. We will use the notation $\left\{Y_{\ell, k}: 1 \leq k \leq N(d, \ell)\right\}$ to denote an orthonormal basis for the eigenspace corresponding to $\lambda_{\ell}$. It is known that this space has dimension

$$
N(d, \ell)= \begin{cases}1, & \text { if } \ell=0 \\
\frac{2 \ell+d-2}{\ell}\left(\begin{array}{c}
\ell+d-3 \\
\ell-1
\end{array}\right), & \text { if } \ell \geq 1\end{cases}
$$

with $N(d, \ell)=\mathcal{O}\left(\ell^{d-2}\right)$ for $\ell \rightarrow \infty$. The collection of all eigenfunctions $Y_{\ell, k}$ forms an orthonormal basis for $L_{2}=L_{2}\left(\mathbb{S}^{d-1}\right)$. This can be used to introduce Sobolev spaces $H^{\sigma}:=\left\{u \in L_{2}:\|u\|_{H^{\sigma}}<\infty\right\}$ with

$$
\|u\|_{H^{\sigma}}^{2}=\sum_{\ell=0}^{\infty} \sum_{k=1}^{N(d, \ell)}\left|\widehat{u}_{\ell, k}\right|^{2}\left(1+\lambda_{\ell}\right)^{\sigma}, \quad \widehat{u}_{\ell, k}=\int_{\mathbb{S}^{d-1}} u(\omega) Y_{\ell, k}(\omega) d \omega .
$$

Obviously, the norm stems from an inner product

$$
\langle u, v\rangle_{H^{\sigma}}=\sum_{\ell=0}^{\infty} \sum_{k=1}^{N(d, \ell)} \widehat{u}_{\ell, k} \widehat{v}_{\ell, k}\left(1+\lambda_{\ell}\right)^{\sigma} .
$$

In the case of a vector-valued function $u: \mathbb{S}^{d-1} \rightarrow \mathbb{R}^{n}$, Sobolev spaces are introduced accordingly, using, for example, the $\ell_{2}$-norm on $\mathbb{R}^{n}$ of the $H^{\sigma}$-norms of the components

$$
\|u\|_{H^{\sigma}}^{2}=\sum_{j=1}^{n}\left\|u_{j}\right\|_{H^{\sigma}}^{2} .
$$

We will not distinguish between vector- and scalar-valued Sobolev spaces. It should become clear from the context which ones are meant.

All this information as well as further material on spherical harmonics can be found in 12 .

1.2. Reproducing Kernel Hilbert spaces. For $\sigma>(d-1) / 2$, the Sobolev embedding theorem guarantees that $H^{\sigma} \subseteq C\left(\mathbb{S}^{d-1}\right)$. Hence, in this situation, $H^{\sigma}$ has a reproducing kernel given by

$$
\Phi_{\sigma}(x, y)=\sum_{\ell=0}^{\infty} \sum_{k=1}^{N(d, \ell)}\left(1+\lambda_{\ell}\right)^{-\sigma} Y_{\ell, k}(x) Y_{\ell, k}(y) .
$$

This kernel is reproducing in the sense that $\Phi_{\sigma}(\cdot, x) \in H^{\sigma}$ for all $x \in \mathbb{S}^{d-1}$ and $u(x)=\left\langle u, \Phi_{\sigma}(\cdot, x)\right\rangle_{H^{\sigma}}$ for all $x \in \mathbb{S}^{d-1}$ and all $u \in H^{\sigma}$. The kernel is also bizonal in the sense that $\Phi_{\sigma}(x, y)=\phi_{\sigma}\left(x^{T} y\right)$ with $\phi_{\sigma}: \mathbb{R} \rightarrow \mathbb{R}$, which immediately follows from the addition theorem for spherical harmonics; see [12]. Obviously, a bizonal kernel is symmetric in the sense that $\Phi(x, y)=\Phi(y, x)$. 
We will relax the idea of a reproducing kernel in the following sense. Suppose, we have a symmetric kernel of the form

$$
\Phi(x, y)=\sum_{\ell=0}^{\infty} \sum_{k=1}^{N(d, \ell)} \widehat{\phi}(\ell) Y_{\ell, k}(x) Y_{\ell, k}(y),
$$

where the Fourier coefficients satisfy

$$
c_{1}\left(1+\lambda_{\ell}\right)^{-\sigma} \leq \widehat{\phi}(\ell) \leq c_{2}\left(1+\lambda_{\ell}\right)^{-\sigma}, \quad \ell \in \mathbb{N}_{0} .
$$

Then, we can introduce a new Hilbert space consisting of all functions $u \in L_{2}\left(\mathbb{S}^{d-1}\right)$ with

$$
\|u\|_{\Phi}^{2}=\sum_{\ell=0}^{\infty} \sum_{k=1}^{N(d, \ell)} \frac{\left|\widehat{u}_{\ell, k}\right|^{2}}{\widehat{\phi}(\ell)}<\infty .
$$

Obviously, this Hilbert space is, because of the decay condition (1.9), algebraically identical with $H^{\sigma}$ and the norm defined by (1.10) is equivalent to the standard norm on $H^{\sigma}$. Furthermore, $\Phi$ is the reproducing kernel of $H^{\sigma}$ with respect to the inner product associated to the norm (1.10). Taking this into account, we will also simply define the norm and inner product on $H^{\sigma}$ to be

$$
\begin{aligned}
\|u\|_{H^{\sigma}}^{2} & =\sum_{\ell=0}^{\infty} \sum_{k=1}^{N(d, \ell)} \frac{\left|\widehat{u}_{\ell, k}\right|^{2}}{\widehat{\phi}(\ell)} \\
\langle u, v\rangle_{H^{\sigma}} & =\sum_{\ell=0}^{\infty} \sum_{k=1}^{N(d, \ell)} \frac{\widehat{u}_{\ell, k} \widehat{v}_{\ell, k}}{\widehat{\phi}(\ell)} .
\end{aligned}
$$

We will use the notation $\|\cdot\|_{\Phi}$ and $\langle\cdot, \cdot\rangle_{\Phi}$ only if it is necessary to distinguish.

This approach gives us access to a variety of kernels, including Sobolev splines and Wendland's compactly supported functions (see [19]). To be more precise, we can use kernels defined on all of $\mathbb{R}^{d}$ and restrict them to $\mathbb{S}^{d-1}$ since it it well known that a kernel $\Psi: \mathbb{R}^{d} \rightarrow \mathbb{R}$, which is the reproducing kernel to $H^{\tau}\left(\mathbb{R}^{d}\right)$ leads via restriction to a kernel $\Phi(x, y)=\Psi(x-y), x, y \in \mathbb{S}^{d-1}$, which is the reproducing kernel of $H^{\tau-1 / 2}$; see 14 .

For vector-valued Sobolev spaces, we will simply use the reproducing kernel theory for each component.

\section{ReACtion-Diffusion EQUATIONS}

In this section, we will shortly discuss the kind of equations we will be analysing. In particular, we are interested in systems (1.1) with initial conditions (1.2).

2.1. Reflections on elliptic operators. In many applications, the elliptic operator $L$ in (1.1) is given by the Laplace-Beltrami operator itself, i.e., $L=-\Delta_{*}$. Here, however, the following differential operator will play a major role in this paper. Let

$$
L=-D \Delta_{*}+\gamma,
$$

where $D$ and $\gamma$ are positive constants in the case of a single equation and are diagonal matrices with positive entries on the diagonal in the case of systems. Obviously, $L$ is a strictly elliptic operator. In principle, it is not necessary that $D$ and $\gamma$ are constants but this assumption will simplify our analysis. Note that, when solving equations (1.1) and (1.2), this particular choice of differential operator is not 
a restriction. For example, the standard heat equation $\partial_{t} u-\Delta_{*} u=0$ can obviously be described in this way, since, with $D=\gamma=1$ and $F(u)=u$, we immediately have $\partial_{t} u+L u=u$. Also, the Allen-Cahn equation $\partial_{t} u-\Delta_{*} u=\frac{1}{\epsilon^{2}} u\left(1-u^{2}\right)$ can be rewritten in the form (1.1) by choosing once again $D=\gamma=1$ and $F(u)=$ $u+\frac{1}{\epsilon^{2}} u\left(1-u^{2}\right)$. The same is true for the Grey-Scott model using

$$
D=\left(\begin{array}{cc}
D_{1} & 0 \\
0 & D_{2}
\end{array}\right), \quad \gamma=\left(\begin{array}{cc}
1 & 0 \\
0 & 1
\end{array}\right), \quad F(u, v)=\left(\begin{array}{c}
-u v^{2}+\widetilde{\gamma}(1-u)+u \\
u v^{2}-(\widetilde{\gamma}+\kappa) v+v
\end{array}\right) .
$$

We will use the fact that if $u$ has Fourier coefficients $\widehat{u}_{\ell, k}$, the function $L u$ has Fourier coefficients

$$
\widehat{(L u)}_{\ell, k}=\left(D \lambda_{\ell}+\gamma\right) \widehat{u}_{\ell, k} .
$$

Consequently,

$$
\begin{aligned}
\|L u\|_{H^{\sigma}}^{2} & =\sum_{\ell=0}^{\infty} \sum_{k=1}^{N(d, \ell)}\left|D \lambda_{\ell}+\gamma\right|^{2}\left|\widehat{u}_{\ell, k}\right|^{2}\left(1+\lambda_{\ell}\right)^{\sigma} \\
& \leq \max \left(D^{2}, \gamma^{2}\right)\|u\|_{H^{\sigma+2}}^{2}
\end{aligned}
$$

and since we can argue similarly for a lower bound, we have the well-known norm equivalence on $H^{\sigma+2}$ given by

$$
k_{1}\|u\|_{H^{\sigma+2}} \leq\|L u\|_{H^{\sigma}} \leq k_{2}\|u\|_{H^{\sigma+2}}
$$

with $k_{1}=\min (D, \gamma)$ and $k_{2}=\max (D, \gamma)$ being independent of the degree of smoothness $\sigma$. Obviously, this remains valid for systems.

Another important consequence is the following. If $\Phi$ is a reproducing kernel of $H^{\sigma}$, then $\Psi(x, y):=L^{(1)} \Phi(x, y)$, where the superscript indicates that the operator $L$ is applied to the first argument of $\Phi$, has a Fourier expansion

$$
\begin{aligned}
\Psi(x, y) & =\sum_{\ell=0}^{\infty} \sum_{k=1}^{N(d, \ell)} \widehat{\phi}(\ell)\left(D \lambda_{\ell}+\gamma\right) Y_{k, \ell}(x) Y_{k, \ell}(y) \\
& =: \sum_{\ell=0}^{\infty} \sum_{k=1}^{N(d, \ell)} \widehat{\psi}(\ell) Y_{k, \ell}(x) Y_{k, \ell}(y) .
\end{aligned}
$$

Hence, $\Psi$ is a zonal kernel, as well, and the norm equivalence (1.9) implies

$$
c_{1}\left(1+\lambda_{\ell}\right)^{-\sigma+1} \leq \widehat{\psi}(\ell) \leq c_{2}\left(1+\lambda_{\ell}\right)^{-\sigma+1} .
$$

We can summarise these simple but important findings as follows.

Lemma 2.1. Let $L$ be the operator $L=-D \Delta_{*}+\gamma I$ with positive constants $D, \gamma$. Then, $L$ induces a norm on $H^{\sigma}, \sigma \geq 2$, via $u \mapsto\|L u\|_{H^{\sigma-2}}$, which is equivalent to the standard norm on $H^{\sigma}$. Furthermore, if $\Phi$ is a reproducing kernel of $H^{\sigma}$, the kernel $\Psi:=L \Phi$ is a reproducing kernel of $H^{\sigma-1}$.

2.2. Semilinear parabolic equations on the sphere. Semilinear parabolic equations are known to have smooth solutions, as long as these solutions exist. To be more precise:

Lemma 2.2. Suppose that $F \in C^{\infty}\left(\mathbb{R}^{n}\right)$ and $u_{0} \in H^{2 \sigma}\left(\mathbb{S}^{d-1}\right)$ with $\sigma \geq 1$. Then there is a time $T>0$ such that (1.1), (1.2) has a unique solution u satisfying

$$
u \in C\left([0, T], H^{2 \sigma}\left(\mathbb{S}^{d-1}\right)\right) \cap C^{1}\left([0, T], H^{2 \sigma-2}\left(\mathbb{S}^{d-1}\right)\right) .
$$


The proof follows essentially from Proposition 1.2 and Proposition 4.2 from Chapter 15 of [16]. The smoothness assumption on the right-hand side $F$ can be further relaxed but is satisfied for what we have in mind.

As usual, we interpret $u$ also as a map $u:[0, T] \rightarrow H^{\sigma}\left(\mathbb{S}^{d-1}\right)$ by defining $u(t)=u(t, \cdot)$ for $t \in[0, T]$. Since $u \in C\left([0, T], H^{\sigma}\left(\mathbb{S}^{d-1}\right)\right.$, there is a finite interval $I=[a, b] \subseteq \mathbb{R}$, such that

$$
\|u(t)\|_{H^{\sigma}} \in I=[a, b], \quad t \in[0, T] .
$$

If $F: \mathbb{R}^{n} \rightarrow \mathbb{R}^{n}$ is a polynomial of degree $m$ such as $F(x)=\sum_{|\alpha| \leq m} c_{\alpha} x^{\alpha}$, then it is easy to see that $\left(D^{\beta} F\right)(u(t)) \in H^{\sigma}$ for $0 \leq|\beta| \leq m$, simply because the triangle inequality and the Cauchy-Schwarz inequality yield for any $\beta \in \mathbb{N}_{0}^{n}$ :

$$
\begin{aligned}
\left\|\left(D^{\beta} F\right)(u(t))\right\|_{H^{\sigma}} & =\left\|\sum_{\substack{\alpha \geq \beta \\
|\alpha| \leq m}} \frac{\alpha !}{(\alpha-\beta) !} c_{\alpha} u^{\alpha-\beta}(t)\right\|_{H^{\sigma}} \\
& \leq \sum_{\substack{\alpha \geq \beta \\
|\alpha| \leq m}} \frac{\alpha !}{(\alpha-\beta) !}\left|c_{\alpha}\right|\|u(t)\|_{H^{\sigma}}^{|\alpha|-|\beta|} \\
& =: C_{\beta}(a, b) .
\end{aligned}
$$

Even more, if we set $I_{\delta}=[a-\delta, b+\delta]$ for $\delta>0$ sufficiently small (i.e. $\delta<(b-a) / 2$ ) and assume that $v(t) \in H^{\sigma}$ with $\|v(t)\|_{H^{\sigma}} \in I_{\delta}$ for $t \in[0, T]$ then

$$
F(v(t))-F(u(t))=\sum_{0<|\alpha| \leq m} \frac{D^{\alpha} F(u(t))}{\alpha !}(v(t)-u(t))^{\alpha}
$$

such that

$$
\begin{aligned}
\|F(u(t))-F(v(t))\|_{H^{\sigma}} & \leq \sum_{0<|\alpha| \leq m} \frac{1}{\alpha !}\left\|D^{\alpha} F(u(t))\right\|_{H^{\sigma}}\|u(t)-v(t)\|_{H^{\sigma}}^{|\alpha|} \\
& \leq\left(\sum_{0<|\alpha| \leq m} \frac{C_{\alpha}(a, b)}{\alpha !}\|u(t)-v(t)\|_{H^{\sigma}}^{|\alpha|-1}\right)\|u(t)-v(t)\|_{H^{\sigma}} \\
& \leq C(a, b, \delta)\|u(t)-v(t)\|_{H^{\sigma}} .
\end{aligned}
$$

For an arbitrary smooth $F$, we can employ Taylor's theorem or use known results on the composition of functions in (fractional) Sobolev spaces (see for example, [3]) to derive the following result.

Lemma 2.3. Suppose $u(t) \in H^{\sigma}, t \in[0, T]$, with $\sigma \geq 2$ satisfies $\|u(t)\|_{H^{\sigma}} \in I=$ $[a, b]$ for $t \in[0, T]$. Suppose $\delta>0$ is given and $I_{\delta}=[a-\delta, b+\delta]$. Let $F \in C^{\infty}\left(\mathbb{R}^{n}\right)$. Then, for any $v \in H^{\sigma}$ we have $F(v) \in H^{\sigma}$ and there is a constant $C_{L}=C(\delta, F, a, b)$ such that

$$
\|F(u(t))-F(v(t))\|_{H^{\sigma}} \leq C_{L}\|u(t)-v(t)\|_{H^{\sigma}}
$$

for all $v \in H^{\sigma}$ with $\|v(t)\|_{H^{\sigma}} \in I_{\delta}$.

Finally, we need the following simple observation.

Lemma 2.4. Let $u \in H^{2 \sigma}$ with $\sigma \geq 2$. Let $L$ be a second-order linear differential operator. Then, $L u \in H^{\sigma}$. 


\section{THE DisCRETISATION SCHEME}

We will use a meshfree approximation method to approximate the solution of (1.1) and (1.2) in space yielding a semi-discrete solution. This method can be interpreted as a collocation method as well as a Petrov-Galerkin method.

We will restrict our description to the scalar-valued case $n=1$ but it should be apparent that systems of equations can be handled in the same manner.

3.1. Collocation. Let $X=\left\{x_{1}, \ldots, x_{N}\right\} \subseteq \mathbb{S}^{d-1}$ be the set of collocation points. Let $\Phi: \mathbb{S}^{d-1} \times \mathbb{S}^{d-1} \rightarrow \mathbb{R}$ be a reproducing kernel of $H^{\sigma}$ with $\sigma \geq \max (2,(d-1) / 2)$. Then, we define the discrete approximation space

$$
V_{X}:=\operatorname{span}\left\{\Phi\left(\cdot, x_{1}\right), \ldots, \Phi\left(\cdot, x_{N}\right)\right\} .
$$

We will now describe an alternative basis for $V_{X}$ consisting of cardinal functions $\psi_{j} \in V_{X}, 1 \leq j \leq N$, i.e., functions satisfying $\psi_{j}\left(x_{k}\right)=\delta_{j k}$.

Lemma 3.1. The cardinal functions $\psi_{j} \in V_{X}$ can be determined by solving the linear system

$$
\left(\begin{array}{ccc}
\Phi\left(x_{1}, x_{1}\right) & \ldots & \Phi\left(x_{1}, x_{N}\right) \\
\vdots & \vdots & \vdots \\
\Phi\left(x_{N}, x_{1}\right) & \ldots & \Phi\left(x_{N}, x_{N}\right)
\end{array}\right)\left(\begin{array}{c}
\psi_{1}(x) \\
\vdots \\
\psi_{N}(x)
\end{array}\right)=\left(\begin{array}{c}
\Phi\left(x_{1}, x\right) \\
\vdots \\
\Phi\left(x_{N}, x\right)
\end{array}\right) .
$$

They obviously form a basis of $V_{X}$.

Proof. This is a standard result on interpolation with radial basis functions and follows from the fact that the matrix in (3.1) is positive definite, hence the vector $\psi(x) \in \mathbb{R}^{N}$ is well defined for all $x \in \mathbb{S}^{d-1}$. Moreover, for $x=x_{k}$, row $i$ of (3.1) becomes

$$
\sum_{j=1}^{N} \Phi\left(x_{i}, x_{j}\right) \psi_{j}\left(x_{k}\right)=\Phi\left(x_{i}, x_{k}\right)
$$

and uniqueness gives $\psi_{j}\left(x_{k}\right)=\delta_{j k}$. Obviously, $\psi_{j} \in V_{X}$ by construction.

With these cardinal functions at hand, we build our approximation $u_{h}(t, \cdot) \in V_{X}$ as

$$
u_{h}(t, x)=\sum_{j=1}^{N} \alpha_{j}(t) \psi_{j}(x)
$$

where the coefficients $\alpha_{j}$ have to be determined. This is done, as usual for such a method-of-line approach, by realising that $u_{h}\left(t, x_{k}\right)=\alpha_{k}(t)$ and hence, the PDE, when restricted to the collocation points becomes an explicit system of ordinary differential equations for the coefficient vector:

$$
\begin{aligned}
\frac{d}{d t} \alpha_{k}(t) & =\partial_{t} u_{h}\left(t, x_{k}\right)=-L u_{h}\left(t, x_{k}\right)+F\left(u_{h}\left(t, x_{k}\right)\right) \\
& =-\sum_{j=1}^{N} \alpha_{j}(t) L \psi_{j}\left(x_{k}\right)+F\left(\sum_{j=1}^{N} \alpha_{j}(t) \psi_{j}\left(x_{k}\right)\right) \\
& =-\sum_{j=1}^{N} \alpha_{j}(t) L \psi_{j}\left(x_{k}\right)+F\left(\alpha_{k}(t)\right)
\end{aligned}
$$


Together with the initial conditions $u_{h}\left(t, x_{k}\right)=u_{0}\left(x_{k}\right)$, this can be written in matrix form as

$$
\begin{aligned}
\frac{d}{d t} \alpha(t) & =B \alpha(t)+F(\alpha(t)), \\
\alpha(0) & =u_{0},
\end{aligned}
$$

where $B=\left(-L \psi_{j}\left(x_{i}\right)\right), F(\alpha)=\left(F\left(\alpha_{i}\right)\right)$, and $u_{0}=\left(u_{0}\left(x_{i}\right)\right)$. Note that the nonlinearity on the right-hand side is rather easily resolved since the system of ordinary differential equations is explicit and the nonlinearity only appears on the right-hand side, again.

3.2. Petrov-Galerkin approximation. We are now going to interpret the above approximation scheme as a Galerkin scheme in $H^{\sigma}$. This will be quite important when it comes to analysing the stability and the approximation error.

To this end, we have to make sure that our approximant $u_{h}=\sum \alpha_{j} \psi_{j}$ satisfies $L u_{h} \in H^{\sigma}$. Obviously, it suffices to look at functions of the form $\Phi_{y}(x)=\Phi(x, y)$ with $y \in \mathbb{S}^{d-1}$ fixed.

Lemma 3.2. Fix $y \in \mathbb{S}^{d-1}$ and define $\Phi_{y}(x)=\Phi(x, y)$, where $\Phi$ is a reproducing kernel of $H^{\sigma}$. Then, $\Phi_{y} \in H^{\tau}$ for all $\tau<2 \sigma-(d-1) / 2$. This means that $L \Phi_{y} \in H^{\sigma}$ if $\sigma>\frac{d-1}{2}+2$.

Proof. For the first part note that (1.8) implies that $\Phi_{y}$ has Fourier coefficients $\left(\widehat{\Phi_{y}}\right)_{\ell, k}=\widehat{\phi}(\ell) Y_{\ell, k}(y)$. Hence, $\Phi_{y} \in H^{\tau}$, if the sum

$$
\begin{aligned}
\left\|\Phi_{y}\right\|_{H^{\tau}}^{2} & =\sum_{\ell=0}^{\infty} \sum_{k=1}^{N(d, \ell)}\left|\left(\widehat{\Phi_{y}}\right)_{\ell, k}\right|^{2}\left(1+\lambda_{\ell}\right)^{\tau} \\
& \leq C \sum_{\ell=0}^{\infty} \sum_{k=1}^{N(d, \ell)}\left(1+\lambda_{\ell}\right)^{-2 \sigma+\tau}\left|Y_{\ell, k}(y)\right|^{2} \\
& =C \sum_{\ell=0}^{\infty} \sum_{k=1}^{N(d, \ell)}\left(1+\lambda_{\ell}\right)^{-2 \sigma+\tau} \frac{N(d, \ell)}{\omega_{d-1}} P_{\ell}(d ; 1) \\
& \leq C \sum_{\ell=0}^{\infty}\left(1+\lambda_{\ell}\right)^{-2 \sigma+\tau} \ell^{d-2} \\
& \leq C \sum_{\ell=0}^{\infty}\left(1+\ell^{2}\right)^{-2 \sigma+\tau+\frac{d}{2}-1}
\end{aligned}
$$

is finite. Here, we have used the addition theorem for spherical harmonics, where $P_{\ell}(d ; \cdot)$ denotes the generalised Legendre polynomials with $\left|P_{\ell}(d ; t)\right| \leq 1$ for all $t \in[-1,1]$, and the fact that $N(d, \ell)=\mathcal{O}\left(\ell^{d-2}\right)$ and $\lambda_{\ell}=\mathcal{O}\left(\ell^{2}\right)$. This sum is obviously finite if $2\left(-2 \sigma+\tau+\frac{d}{2}-1\right)<-1$, which equivalently means $\tau<2 \sigma-\frac{d-1}{2}$.

The second part follows since $L$ is a second-order operator, such that $L \Phi_{y} \in$ $H^{\tau-2} \subseteq H^{\sigma}$ if $\sigma \leq \tau-2<2 \sigma-\frac{d-1}{2}-2$, which resolves to $\sigma>\frac{d-1}{2}+2$.

On account of this result, we will, from now on, assume that $\sigma>2+(d-1) / 2$.

The usual strategy for a Galerkin approximation is to multiply equation (1.1) with a test function $\chi$ and then to integrate the new equation. This leads to

$$
\left\langle\partial_{t} u_{h}, \chi\right\rangle+\left\langle L u_{h}, \chi\right\rangle=\left\langle F\left(u_{h}\right), \chi\right\rangle \text {. }
$$


If we just integrate then the inner product in the above equation is just the $L_{2}$ inner product on the sphere. The next step would be to integrate the term $\langle L u, \chi\rangle$ by parts and to restrict $\chi$ to our discretisation space $V_{X}$.

However, we will go a slightly different way. Instead of using the $L_{2}$ inner product, we will use the $H^{\sigma}$ inner product induced by the kernel $\Phi$. We will also not integrate by parts but still restrict the choice of $\chi$ to $V_{X}$, i.e., we are looking at the equation

$$
\left\langle\partial_{t} u_{h}, \chi\right\rangle_{H^{\sigma}}=-\left\langle L u_{h}, \chi\right\rangle_{H^{\sigma}}+\left\langle F\left(u_{h}\right), \chi\right\rangle_{H^{\sigma}}, \quad \chi \in V_{X} .
$$

If we use the expansion (3.2) for $u_{h}$ in terms of the cardinal functions $\psi_{j}$ and test with the basis function $\Phi\left(\cdot, x_{k}\right)$, we derive a Petrov-Galerkin approximation of the form

$$
\begin{aligned}
& \sum_{j=1}^{N} \alpha_{j}^{\prime}(t)\left\langle\psi_{j}, \Phi\left(\cdot, x_{k}\right)\right\rangle_{H^{\sigma}} \\
& \quad=-\sum_{j=1}^{N} \alpha_{j}(t)\left\langle L \psi_{j}, \Phi\left(\cdot, x_{k}\right)\right\rangle_{H^{\sigma}}+\left\langle F\left(\sum_{j=1}^{N} \alpha_{j}(t) \psi_{j}\right), \Phi\left(\cdot, x_{k}\right)\right\rangle_{H^{\sigma}} .
\end{aligned}
$$

However, using the reproducing kernel property, we can see that the mass and stiffness matrix and the right-hand side are actually given by

$$
\begin{aligned}
\left\langle\psi_{j}, \Phi\left(\cdot, x_{k}\right)\right\rangle_{H^{\sigma}} & =\psi_{j}\left(x_{k}\right)=\delta_{j k}, \\
-\left\langle L \psi_{j}, \Phi\left(\cdot, x_{k}\right)\right\rangle_{H^{\sigma}} & =-L \psi_{j}\left(x_{k}\right), \\
\left\langle F\left(\sum_{j=1}^{N} \alpha_{j}(t) \psi_{j}\right), \Phi\left(\cdot, x_{k}\right)\right\rangle_{H^{\sigma}} & =F\left(\sum_{j=1}^{N} \alpha_{j}(t) \psi_{j}\left(x_{k}\right)\right)=F\left(\alpha_{k}(t)\right) .
\end{aligned}
$$

Hence, we have recovered the collocation scheme from the previous section.

Corollary 3.3. The $H^{\sigma}$-Petrov-Galerkin method (3.3) with trial functions $\psi_{j}$ and test functions $\Phi\left(\cdot, x_{j}\right)$ is identical with the collocation method from Section 3.1 .

\section{Analysis of the SEMI-DisCRETE SCHEMe}

4.1. Stability. We will now show that the collocation scheme is stable for finite time intervals. To this end, we will, as usual, use Gronwall's inequality in the form that if $u$ is continuous on $[0, T]$ and $h$ is integrable on $[0, T]$ and nonnegative, then an estimate of the form

$$
u(t) \leq K+\int_{0}^{t} h(s) u(s) d s, \quad t \in[0, T]
$$

with a constant $K$ implies

$$
u(t) \leq K \exp \left(\int_{0}^{t} h(s) d s\right) .
$$

For our proof it will be essential that the collocation solution is also the PetrovGalerkin solution in $H^{\sigma}$ with reproducing kernel $\Phi$, as described above.

Theorem 4.1. Let $u_{0} \in H^{2 \sigma}$ with $\sigma>(d-1) / 2+2$. There is a constant $C=C_{T}$ such that the semi-discrete solution $u_{h}(t)$ satisfies

$$
\left\|u_{h}(t)\right\|_{H^{\sigma}} \leq C_{T}\left(\left\|u_{h}(0)\right\|_{H^{\sigma}}+1\right) .
$$


Proof. We will prove this only under the assumption that $F$ is globally Lipschitz continuous in the sense that (2.2) holds for all $v \in H^{\sigma}$. The general case will follow using the same arguments as in the following proof of Theorem 4.5.

Since, by our assumptions, $\partial_{t} u_{h}(t, \cdot), L u_{h}(t, \cdot)$ and $F\left(u_{h}(t, \cdot)\right)$ all belong to $H^{\sigma}$, we know that the collocation equations

$$
\partial_{t} u_{h}\left(t, x_{k}\right)=-L u_{h}\left(t, x_{k}\right)+F\left(u_{h}\left(t, x_{k}\right)\right), \quad 1 \leq k \leq N,
$$

are equivalent to (3.3). Hence, using the fact that $u_{h}(t) \in V_{X}$, we can derive

$$
\left\langle\partial_{t} u_{h}(t), u_{h}(t)\right\rangle_{H^{\sigma}}=-\left\langle L u_{h}(t), u_{h}(t)\right\rangle_{H^{\sigma}}+\left\langle F\left(u_{h}(t)\right), u_{h}(t)\right\rangle_{H^{\sigma}},
$$

which leads to

$$
\begin{aligned}
\frac{1}{2} \frac{d}{d t}\left\|u_{h}(t)\right\|_{H^{\sigma}}^{2} & =-\left\langle L u_{h}(t), u_{h}(t)\right\rangle_{H^{\sigma}}+\left\langle F\left(u_{h}(t)\right), u_{h}(t)\right\rangle_{H^{\sigma}} \\
& \leq\left\|F\left(u_{h}(t)\right)\right\|_{H^{\sigma}}\left\|u_{h}(t)\right\|_{H^{\sigma}}
\end{aligned}
$$

using the fact that $L$ is a positive operator. Next, our assumption on the Lipschitz continuity of $F$ guarantees that

$$
\begin{aligned}
\left\|F\left(u_{h}(t)\right)\right\|_{H^{\sigma}} & \leq\left\|F\left(u_{h}(t)\right)-F(0)\right\|_{H^{\sigma}}+\|F(0)\|_{H^{\sigma}} \\
& \leq C_{L}\left\|u_{h}(t)\right\|_{H^{\sigma}}+C .
\end{aligned}
$$

Inserting this into the previous estimate and using

gives

$$
\frac{d}{d t}\left\|u_{h}(t)\right\|_{H^{\sigma}}^{2}=2\left\|u_{h}(t)\right\|_{H^{\sigma}} \frac{d}{d t}\left\|u_{h}(t)\right\|_{H^{\sigma}}
$$

$$
\frac{d}{d t}\left\|u_{h}(t)\right\|_{H^{\sigma}} \leq C\left(\left\|u_{h}(t)\right\|_{H^{\sigma}}+1\right) .
$$

Integrating this inequality over $[0, t] \subseteq[0, T]$ then gives

$$
\begin{aligned}
\left\|u_{h}(t)\right\|_{H^{\sigma}} & \leq\left\|u_{h}(0)\right\|_{H^{\sigma}}+C \int_{0}^{t}\left(1+\left\|u_{h}(s)\right\|_{H^{\sigma}}\right) d s \\
& \leq\left\|u_{h}(0)\right\|_{H^{\sigma}}+C T+C \int_{0}^{t}\left\|u_{h}(s)\right\|_{H^{\sigma}} d s .
\end{aligned}
$$

Thus, Gronwall's inequality yields finally,

$$
\begin{aligned}
\left\|u_{h}(t)\right\|_{H^{\sigma}} & \leq\left(\left\|u_{h}(0)\right\|_{H^{\sigma}}+C T\right) \exp \left(\int_{0}^{t} C d s\right) \\
& \leq e^{C T}\left(C T+\left\|u_{h}(0)\right\|_{H^{\sigma}}\right) \\
& =: C_{T}\left(\left\|u_{h}(0)\right\|_{H^{\sigma}}+1\right),
\end{aligned}
$$

which is the desired estimate.

4.2. Error analysis. We will now analyse the error of the semi-discrete solution. We will again employ the equivalent form as a Petrov-Galerkin scheme and follow ideas from [18].

Error estimates will be stated using the fill distance

$$
h=h_{X}=\max _{x \in \mathbb{S}^{d-1}} \min _{x_{j} \in X} \operatorname{dist}\left(x, x_{j}\right),
$$

which measures the radius of the largest spherical cap containing no data site. The following lemma states the well-known forward estimates for scattered data approximation on spheres (cf. [13, 10]). 
Lemma 4.2. Let $X \subseteq \mathbb{S}^{d-1}$ have a sufficiently small fill distance $h_{X}$. Let $\sigma>$ $(d-1) / 2$ be given. Then, for every function $u \in H^{\sigma}$ with $u \mid X=0$, and every $0 \leq \beta \leq \sigma$, there is a constant $C>0$ independent of $u$ and $X$ such that

$$
\|u\|_{H^{\beta}} \leq C h_{X}^{\sigma-\beta}\|u\|_{H^{\sigma}} .
$$

Next, we need a construction, which mimics the Ritz projection in classical finite elements.

Theorem 4.3. Let $\sigma>(d-1) / 2+2$. There is a linear operator $R_{h}: H^{2 \sigma} \rightarrow V_{X}$ with $L R_{h} u\left(x_{k}\right)=L u\left(x_{k}\right)$ for $1 \leq k \leq N$. Furthermore, For $2 \leq \beta \leq \sigma+1$, we have the estimate

$$
\left\|R_{h} u-u\right\|_{H^{\beta}} \leq C h_{X}^{2 \sigma-\beta}\|u\|_{H^{2 \sigma}}
$$

and hence, in particular,

$$
\left\|R_{h} u-u\right\|_{H^{\sigma}} \leq C h_{X}^{\sigma}\|u\|_{H^{2 \sigma}} .
$$

Proof. We define $R_{h} u(x)=\sum_{j=1}^{N} \alpha_{j} \Phi\left(x, x_{j}\right)$ where the coefficients are determined by the interpolation conditions

$$
L R_{h} u\left(x_{i}\right)=\sum_{j=1}^{N} \alpha_{j} L^{(1)} \Phi\left(x_{i}, x_{j}\right)=L u\left(x_{i}\right), \quad 1 \leq i \leq N .
$$

Here, the upper index indicates that the elliptic operator is applied with respect to the first argument of the kernel. Since $\Phi$ is a reproducing kernel of $H^{\sigma}$, we know that $\Psi=L \Phi$ is a reproducing kernel of $H^{\sigma-1}$. Particularly, the kernel is also positive definite, showing that the above system is uniquely solvable. Furthermore, we see that the interpolant based on $X$ and $\Psi$ to $f=L u$ is given by $I_{X} f=L R_{h} u$. Thus, standard RBF interpolation yields orthogonality

$$
\left\langle L u-L R_{h} u, \Psi\left(\cdot, x_{j}\right)\right\rangle_{\Psi}=0, \quad 1 \leq j \leq N,
$$

and thus

$$
\left\langle L u-L R_{h} u, L R_{h} u\right\rangle_{\Psi}=0 .
$$

This leads to

$$
\begin{aligned}
\left\|L u-L R_{h} u\right\|_{\Psi}^{2} & =\left\langle L u-L R_{h} u, L u\right\rangle_{\Psi} \\
& =\sum_{\ell=0}^{\infty} \sum_{k=1}^{N(d, \ell)} \frac{\left(D \lambda_{\ell}+\gamma\right)}{\widehat{\phi}(\ell)} \widehat{u}_{\ell, k}\left(\widehat{u}_{\ell, k}-\widehat{R}_{h} u_{\ell, k}\right) \\
& \leq\left(\sum_{\ell, k}\left|\widehat{u}_{\ell, k}\right|^{2} \widehat{\phi}(\ell)^{-2}\right)^{1 / 2}\left(\sum_{\ell, k}\left(D \lambda_{\ell}+\gamma\right)^{2}\left|\widehat{u}_{\ell, k}-\widehat{R}_{h} u_{\ell, k}\right|^{2}\right)^{1 / 2} \\
& \leq C\|u\|_{H^{2 \sigma}}\left\|L u-L R_{h} u\right\|_{L_{2}} .
\end{aligned}
$$

Next, Lemma 4.2 yields with $h=h_{X}$ that

$$
\left\|L u-L R_{h} u\right\|_{L_{2}} \leq C h^{\sigma-1}\left\|L u-L R_{h} u\right\|_{H^{\sigma-1}}=C h^{\sigma-1}\left\|u-R_{h} u\right\|_{H^{\sigma+1}}
$$

such that

$$
\left\|u-R_{h} u\right\|_{H^{\sigma+1}}^{2} \leq C\left\|L u-L R_{h} u\right\|_{\Psi}^{2} \leq C h^{\sigma-1}\left\|u-R_{h} u\right\|_{H^{\sigma+1}}\|u\|_{H^{2 \sigma}}
$$

or

$$
\left\|u-R_{h} u\right\|_{H^{\sigma+1}} \leq C h^{\sigma-1}\|u\|_{H^{2 \sigma}} .
$$


Inserting this back into (4.1) yields

$$
\left\|u-R_{h} u\right\|_{H^{2}} \leq C\left\|L u-L R_{h} u\right\|_{L_{2}} \leq C h^{2 \sigma-2}\|u\|_{H^{2 \sigma}} .
$$

Finally, standard interpolation theory in Sobolev spaces applied to (4.2) and (4.3) gives

$$
\left\|u-R_{h} u\right\|_{H^{\beta}} \leq C h^{2 \sigma-\beta}\|u\|_{H^{\beta}},
$$

for every $2 \leq \beta \leq \sigma+1$.

Remark 4.4. Interpolation between (4.2) and $\left\|u-R_{h} u\right\|_{H^{\sigma+1}} \leq C\|u\|_{H^{\sigma+1}}$ shows that we also have

$$
\left\|u-R_{h} u\right\|_{H^{\sigma+1}} \leq C h^{\gamma-\sigma-1}\|u\|_{H^{\gamma}}
$$

for all $u \in H^{\gamma}$ with $\sigma+1 \leq \gamma \leq 2 \sigma$.

After this, we are in the position to state and prove our main result for the semi-discrete approximation.

Theorem 4.5. Let $u_{0} \in H^{2 \sigma}$ with $\sigma>(d-1) / 2+2$. There is a constant $C=C_{T}$ such that the error between the semi-discrete approximation $u_{h}(t)$ and the solution $u(t)$ of (1.1), (1.2) can be bounded by

$$
\left\|u(t)-u_{h}(t)\right\|_{H^{\sigma}} \leq C h^{\sigma}\left(\left\|u_{0}\right\|_{H^{2 \sigma}}+\|u\|_{H^{1}\left([0, T] ; H^{2 \sigma}\right)}\right) .
$$

Proof. First, we will assume that $F$ is globally Lipschitz continuous in the sense that (2.2) holds for all $v \in H^{\sigma}$.

As usual in this context, we write $u_{h}-u=\left(u_{h}-R_{h} u\right)+\left(R_{h} u-u\right)=: \theta+\rho$. From Theorem 4.3 we know that, for $t \in[0, T]$,

$$
\begin{aligned}
\|\rho(t)\|_{H^{\sigma}} & \leq C h^{\sigma}\|u(t)\|_{H^{2 \sigma}} \\
& \leq C h^{\sigma}\left\{\left\|u_{0}\right\|_{H^{2 \sigma}}+\int_{0}^{T}\left\|u_{t}(s)\right\|_{H^{2 \sigma}} d s\right\} .
\end{aligned}
$$

In the following, we will suppress the variable $t$, whenever possible. To estimate the norm of $\theta$, we observe that for every $\chi \in V_{X}$ we have

$$
\begin{aligned}
\left\langle\theta_{t}, \chi\right\rangle_{H^{\sigma}}+\langle L \theta, \chi\rangle_{H^{\sigma}} & =\left\langle\partial_{t} u_{h}+L u_{h}, \chi\right\rangle_{H^{\sigma}}-\left\langle\partial_{t} R_{h} u+L R_{h} u, \chi\right\rangle_{H^{\sigma}} \\
& =\left\langle F\left(u_{h}\right), \chi\right\rangle_{H^{\sigma}}-\left\langle\partial_{t} R_{h} u+L u, \chi\right\rangle_{H^{\sigma}} \\
& =\left\langle F\left(u_{h}\right), \chi\right\rangle_{H^{\sigma}}-\left\langle\partial_{t} R_{h} u+F(u)-\partial_{t} u, \chi\right\rangle_{H^{\sigma}} \\
& =\left\langle F\left(u_{h}\right)-F(u), \chi\right\rangle_{H^{\sigma}}-\left\langle\rho_{t}, \chi\right\rangle_{H^{\sigma}} .
\end{aligned}
$$

Setting $\chi=\theta$ yields

$$
\begin{aligned}
\frac{1}{2} \frac{d}{d t}\|\theta\|_{H^{\sigma}}^{2} & =-\langle L \theta, \theta\rangle_{H^{\sigma}}+\left\langle F\left(u_{h}\right)-F(u), \theta\right\rangle_{H^{\sigma}}-\left\langle\rho_{t}, \theta\right\rangle_{H^{\sigma}} \\
& \leq\left\|F\left(u_{h}\right)-F(u)\right\|_{H^{\sigma}}\|\theta\|_{H^{\sigma}}+\left\|\rho_{t}\right\|_{H^{\sigma}}\|\theta\|_{H^{\sigma}} \\
& \leq\left\{C_{L}\left\|u_{h}-u\right\|_{H^{\sigma}}+\left\|\rho_{t}\right\|_{H^{\sigma}}\right\}\|\theta\|_{H^{\sigma}} .
\end{aligned}
$$

Since $\frac{1}{2} \frac{d}{d t}\|\theta\|_{H^{\sigma}}^{2}=\|\theta\|_{H^{\sigma}} \frac{d}{d t}\|\theta\|_{H^{\sigma}}$ we can conclude, using also the bounds on $\rho$ and $\rho_{t}$, that

$$
\begin{aligned}
\frac{d}{d t}\|\theta\|_{H^{\sigma}} & \leq C{ }_{L}\left\|u_{h}-u\right\|_{H^{\sigma}}+\left\|\rho_{t}\right\|_{H^{\sigma}} \\
& \leq C\left[\|\theta\|_{H^{\sigma}}+\|\rho\|_{H^{\sigma}}+\left\|\rho_{t}\right\|_{H^{\sigma}}\right] \\
& \leq C\|\theta\|_{H^{\sigma}}+C h^{\sigma}\left[\|u\|_{H^{2 \sigma}}+\left\|\partial_{t} u\right\|_{H^{2 \sigma}}\right] .
\end{aligned}
$$


Integrating this inequality leads to

$$
\begin{aligned}
\|\theta(t)\|_{H^{\sigma}} \leq & \|\theta(0)\|_{H^{\sigma}}+C h^{\sigma} \int_{0}^{T}\left[\|u(s)\|_{H^{2 \sigma}}+\left\|\partial_{t} u(s)\right\|_{H^{2 \sigma}}\right] d s \\
& +\int_{0}^{t} C\|\theta(s)\|_{H^{\sigma}} d s,
\end{aligned}
$$

such that Gronwall's lemma leads to

$$
\|\theta(t)\|_{H^{\sigma}} \leq C_{T}\left[\|\theta(0)\|_{H^{\sigma}}+h^{\sigma} \int_{0}^{T}\left(\|u(s)\|_{H^{2 \sigma}}+\left\|\partial_{t} u(s)\right\|_{H^{2 \sigma}}\right) d s\right] .
$$

The initial error $\theta(0)$ can be bounded via

$$
\begin{aligned}
\|\theta(0)\|_{H^{\sigma}} & =\left\|u_{h}(0)-R_{h} u(0)\right\|_{H^{\sigma}} \leq\left\|u_{h}(0)-u(0)\right\|_{H^{\sigma}}+\left\|u(0)-R_{h} u(0)\right\|_{H^{\sigma}} \\
& \leq C h^{\sigma}\|u(0)\|_{H^{2 \sigma}}+C h^{\sigma}\|u(0)\|_{H^{2 \sigma}},
\end{aligned}
$$

where the first bound comes from the fact that $u_{h}(0)$ is the interpolant from $V_{X}$ to $u_{0}$. Thus, we have

$$
\|\theta(t)\|_{H^{\sigma}} \leq C h^{\sigma}\left(\|u(0)\|_{H^{2 \sigma}}+\int_{0}^{T}\left(\|u(s)\|_{H^{2 \sigma}}+\left\|\partial_{t} u(s)\right\|_{H^{2 \sigma}}\right) d s\right) .
$$

This, together with the bound on $\rho$ gives the stated result in the case that $F$ is globally Lipschitz continuous.

If $F$ is not globally Lipschitz continuous, then we have to modify the proof as follows.

First note that by Lemma 2.3. we know that $\|u(t)\|_{H^{\sigma}} \in I=[a, b]$ for $t \in[0, T]$ for a certain interval $I=[a, b]$. Furthermore, if we fix a $\delta \in(0,(b-a) / 2)$, then we know that $F$ is at least locally Lipschitz continuous in the sense of Lemma 2.3. The proof so far remains valid if we can show that $\left\|u_{h}(t)\right\|_{H^{\sigma}} \in I_{\delta}$ for every $t \in[0, T]$, provided $h$ is sufficiently small.

To see this, we pick an $h_{1}$ such that for $h \leq h_{1}$ we find

$$
\|\rho(t)\|_{H^{\sigma}}=\left\|R_{h} u(t)-u(t)\right\|_{H^{\sigma}} \leq C h^{\sigma}\|u(t)\|_{H^{2 \sigma}}<\delta / 2 .
$$

This is possible because of Theorem 4.3 and because Lemma 2.2 implies that even $\|u(t)\|_{H^{2 \sigma}}$ is uniformly bounded in $t \in[0, T]$. Thus, with $I_{\delta}=[a-\delta, b+\delta]$, we have, in particular, that $\left\|R u_{h}(t)\right\|_{H^{\sigma}} \in I_{\delta / 2}$ for $t \in[0, T]$.

Next, define for such an $h \leq h_{1}$ the time $t_{h}=\sup \left\{t \in[0, T]:\left\|u_{h}(t)\right\|_{H^{\sigma}} \in I_{\delta}\right\}$. We must have $t_{h}>0$ since $u_{h}(0)$ is simply the interpolant to $u_{0}$ and satisfies a bound like

$$
\left\|u_{h}(0)-u_{0}\right\|_{H^{\sigma}} \leq C h^{\sigma}\left\|u_{0}\right\|_{H^{2 \sigma}} \leq \delta .
$$

For any $t \leq t_{h}$ we then obviously have $\left\|u_{h}(t)\right\|_{H^{\sigma}} \in I_{\delta}$ and we wish to show that $t_{h}=T$. We can use the local Lipschitz continuity of $F$ such that the analysis of the first part of this proof applies, showing

$$
\|\theta(t)\|_{H^{\sigma}} \leq C_{T} h^{\sigma}\left(\|u(0)\|_{H^{2 \sigma}}+\|u\|_{H^{1}\left([0, T], H^{2 \sigma}\right)}\right)<\delta / 2,
$$

where the last inequality holds for given $h \leq h_{2}$. Hence, for any $t \leq t_{h}$ with $h \leq \min \left(h_{1}, h_{2}\right)$ we have

$$
\left\|u(t)-u_{h}(t)\right\|_{H^{\sigma}} \leq\|\rho(t)\|_{H^{\sigma}}+\|\theta(t)\|_{H^{\sigma}}<\delta,
$$

which means $\left\|u_{h}(t)\right\|_{H^{\sigma}} \in I_{\delta}$. Continuity now shows that we could choose $t_{h}$ even slightly larger. However, we have chosen $t_{h} \leq T$ as the maximum time with the 
property $\|u(t)\|_{H^{\sigma}} \in I_{\delta}$ for all $t \leq t_{h}$. This actually means $t_{h}=T$ and thus the first part of the proof applies. This concludes the proof.

\section{Discretisation in time}

We will now discuss discretisation in time. We will give two examples of implicit discretisation schemes, implicit Euler and linearised Crank-Nicolson. We will explicitly prove convergence and stability of the Crank-Nicolson method. The proof is meant as a model proof for higher order discretisation methods. There is obviously no restriction to these time discretisations and the high convergence order in space would suggest to use also higher order discretisations in time. However, this is not the goal of this paper. Analysing convergence of a larger class of time discretisations would be beyond the scope of this paper.

We have to discretise the nonlinear system of ordinary differential equations

$$
\begin{aligned}
\frac{d}{d t} \alpha(t) & =B \alpha(t)+F(\alpha(t)), \\
\alpha(0) & =u_{0},
\end{aligned}
$$

where $B=\left(-L \psi_{j}\left(x_{i}\right)\right), F(\alpha)=\left(F\left(\alpha_{i}\right)\right)$, and $u_{0}=\left(u_{0}\left(x_{i}\right)\right)$. A first important remark concerns the matrix $B$.

Lemma 5.1. The matrix $B=\left(-L \psi_{j}\left(x_{i}\right)\right)$ is negative definite.

Proof. The vector $\psi(x)$ of the cardinal functions $\psi_{j}(x)$ is given by

$$
\psi(x)=A^{-1} R(x),
$$

where $A=\left(\Phi\left(x_{i}, x_{j}\right)\right)$ is a positive definite, symmetric matrix and $R(x)$ is the vector with entries $R_{i}(x)=\Phi\left(x_{i}, x\right)$. Thus, we have $-L \psi(x)=-A^{-1} L R(x)$. Evaluating at $x=x_{i}$ gives the vector $b_{i}=-A^{-1} L R\left(x_{i}\right)$ such that $B$ can be written as

$$
\begin{aligned}
B & =\left(\begin{array}{c}
b_{1}^{T} \\
\vdots \\
b_{N}^{T}
\end{array}\right)=-\left(\begin{array}{c}
(L R)^{T}\left(x_{1}\right) A^{-1} \\
\vdots \\
(L R)^{T}\left(x_{N}\right) A^{-1}
\end{array}\right)=-\left(\begin{array}{ccc}
L \Phi\left(x_{1}, x_{1}\right) & \ldots & L \Phi\left(x_{N}, x_{1}\right) \\
\vdots & & \vdots \\
L \Phi\left(x_{N}, x_{1}\right) & \ldots & L \Phi\left(x_{N}, x_{N}\right)
\end{array}\right) A^{-1} \\
& =:-A_{L} A^{-1} .
\end{aligned}
$$

Since $A$ is positive definite and $L$ is an elliptic operator, which means that $A_{L}$ is also positive definite, we see that $B$ is negative definite.

Throughout this section we will assume that we work on a fixed time interval $[0, T]$ and that we discretise this time interval using equally spaced time discretisation points $t_{n}=n \tau$ for $0 \leq n \leq N_{T}$ with $\tau=T / N_{T}$.

For completeness, we start with an implicit Euler scheme for (5.1), though the order will only be linear in time and hence the method is not really suited for such a high-order method in space. Generalisations to more sophisticated time-stepping procedures are of course possible and we will discuss the linearised Crank-Nicolson scheme as an example.

Implicit or backward Euler for (5.1) is given by

$$
\frac{\alpha^{n}-\alpha^{n-1}}{\tau}=B \alpha^{n}+F\left(\alpha^{n}\right) \text {. }
$$


Obviously, the fact that $\alpha^{n}$ appears as an argument of the nonlinear function $F$ causes problems, which are, in this case, most appropriately resolved by looking at the linearised implicit Euler scheme

$$
\frac{\alpha^{n}-\alpha^{n-1}}{\tau}=B \alpha^{n}+F\left(\alpha^{n-1}\right)
$$

which then takes the form

$$
(I-\tau B) \alpha^{n}=\alpha^{n-1}+\tau F\left(\alpha^{n-1}\right) .
$$

Since, by Lemma 5.1, the matrix $B$ is negative definite, this method is unconditionally stable and we can always solve for a new vector $\alpha^{n+1}$. Associated with these coefficient vectors are the approximate solutions

$$
U^{n}=\sum_{j=1}^{N} \alpha_{j}^{n} \psi_{j}
$$

A standard but also technical analysis, which follows a standard analysis of a linearised implicit Euler scheme for classical finite elements simply by replacing $L_{2}$ inner products with $H^{\sigma}$ inner products (see for example [17, Theorem 13.3]) and which we will omit here, shows the following result.

Theorem 5.2. The linearised implicit Euler scheme (5.3) is unconditionally stable. The spatial error between the fully discretised solution $U^{n}$ and the true solution $u\left(t_{n}\right)$ can be bounded by

$$
\begin{aligned}
\left\|U^{n}-u\left(t_{n}\right)\right\|_{H^{\sigma}} \leq & C_{1} h^{\sigma}\left\{\left\|u_{0}\right\|_{H^{2 \sigma}}+\int_{0}^{T}\left\|u_{t}\right\|_{H^{2 \sigma}} d s+\tau \sum_{j=0}^{n}\left\|u\left(t_{j}\right)\right\|_{H^{2 \sigma}}\right\} \\
& +C_{2} \tau \int_{0}^{T}\left\|u_{t t}\right\|_{H^{\sigma}} d s \\
= & C(u)\left(\tau+h^{\sigma}\right) .
\end{aligned}
$$

As an example of a higher-order discretisation in time, we will discuss the linearised implicit Crank-Nicolson method.

Assuming again that the time discretisation is given by $t_{n}=n \tau$, the implicit Crank-Nicolson method for the system (5.1) is given by

$$
\frac{\alpha^{n}-\alpha^{n-1}}{\tau}=B \frac{\alpha^{n}+\alpha^{n-1}}{2}+F\left(\frac{\alpha^{n}+\alpha^{n-1}}{2}\right),
$$

which again suffers from the presence of the next solution $\alpha^{n}$ within the nonlinear function $F$. A typical linearisation of this, based on an extrapolation, is given by

$$
\frac{\alpha^{n}-\alpha^{n-1}}{\tau}=B \frac{\alpha^{n}+\alpha^{n-1}}{2}+F\left(\frac{3}{2} \alpha^{n-1}-\frac{1}{2} \alpha^{n-2}\right),
$$

which results in the scheme

$$
\left(I-\frac{\tau}{2} B\right) \alpha^{n}=\left(I+\frac{\tau}{2} B\right) \alpha^{n-1}+\tau F\left(\frac{3}{2} \alpha^{n-1}-\frac{1}{2} \alpha^{n-2}\right),
$$

which, however, now requires two initial values. We will use the following scheme to acquire the second initial value. It is an obvious modification from a scheme given in [17. 
First, we choose $U^{0} \in V_{X}$ as the interpolant to $u^{0}$ on $X$. Then, we define an intermediate function $U^{1,0} \in V_{X}$ as the solution of

$$
\left\langle\frac{U^{1,0}-U^{0}}{\tau}, \chi\right\rangle_{H^{\sigma}}+\left\langle L \frac{U^{1,0}+U^{0}}{2}, \chi\right\rangle_{H^{\sigma}}=\left\langle F\left(U^{0}\right), \chi\right\rangle_{H^{\sigma}}, \quad \chi \in V_{X}
$$

and then the second initial value $U^{1} \in V_{X}$ via solving

$$
\left\langle\frac{U^{1}-U^{0}}{\tau}, \chi\right\rangle_{H^{\sigma}}+\left\langle L \frac{U^{1}+U^{0}}{2}, \chi\right\rangle_{H^{\sigma}}=\left\langle F\left(\frac{U^{1}+U^{1,0}}{2}\right), \chi\right\rangle_{H^{\sigma}}, \quad \chi \in V_{X} .
$$

This can again be recast as a generalised interpolation problem, which does not require (numerical) integration. The proof of the following theorem borrows ideas from a similar theorem from [17.

Theorem 5.3. The linearised Crank-Nicolson scheme is unconditionally stable. For sufficiently smooth solutions on an interval $[0, T]$, the error between the fully discretised solution $U^{n}$ and the true solution $u\left(t_{n}\right)$ can be bounded by

$$
\left\|U^{n}-u\left(t_{n}\right)\right\|_{H^{\sigma}} \leq C(u)\left(\tau^{2}+h^{\sigma}\right) .
$$

Proof. We will restrict ourselves once again to the case where $F$ is globally Lipschitz continuous. As in this context usual, we will use the following notation. We will write $u^{n}=u\left(t_{n}\right)$ and $w_{h}^{n}=R_{h} u\left(t_{n}\right)$. The time steps are given by $t_{n}=n \tau$ and we will also use $t_{n-1 / 2}=(n-1 / 2) \tau$. Furthermore, we will employ the following operators, not only for the sequence $U^{n}$,

$$
\bar{\partial} U^{n}=\frac{U^{n}-U^{n-1}}{\tau}, \quad \widehat{U}^{n}=\frac{U^{n}+U^{n-1}}{2}, \quad \bar{U}^{n}=\frac{3}{2} U^{n-1}-\frac{1}{2} U^{n-2} .
$$

With this notation, (5.5) can be reformulated as

$$
\left\langle\bar{\partial} U^{n}, \chi\right\rangle_{H^{\sigma}}+\left\langle L \widehat{U}^{n}, \chi\right\rangle_{H^{\sigma}}=\left\langle F\left(\bar{U}^{n}\right), \chi\right\rangle_{H^{\sigma}}, \quad \chi \in V_{X} .
$$

We split the error again in the form

$$
U^{n}-u^{n}=\left(U^{n}-w_{h}^{n}\right)+\left(w_{h}^{n}-u^{n}\right)=\theta^{n}+\rho^{n},
$$

and know from Theorem 4.3 that

$$
\left\|\rho^{n}\right\|_{H^{\sigma}} \leq C h^{\sigma}\left\|u^{n}\right\|_{H^{2 \sigma}} .
$$

For the $\theta^{n}$ part of the error, we first note that for $\chi \in V_{X}$,

$$
\begin{aligned}
\left\langle\bar{\partial} \theta^{n}, \chi\right\rangle_{H^{\sigma}}+\left\langle L \widehat{\theta}^{n}, \chi\right\rangle_{H^{\sigma}} & =\left\langle\bar{\partial} U^{n}, \chi\right\rangle_{H^{\sigma}}+\left\langle L \widehat{U}^{n}, \chi\right\rangle_{H^{\sigma}}-\left\langle\bar{\partial} w_{h}^{n}, \chi\right\rangle_{H^{\sigma}}-\left\langle L \widehat{w}_{h}^{n}, \chi\right\rangle_{H^{\sigma}} \\
& =\left\langle F\left(\bar{U}^{n}\right), \chi\right\rangle_{H^{\sigma}}-\left\langle u_{t}^{n-1 / 2}, \chi\right\rangle_{H^{\sigma}}-\left\langle\bar{\partial} w_{h}^{n}-u_{t}^{n-1 / 2}, \chi\right\rangle_{H^{\sigma}} \\
& -\left\langle L w_{h}^{n-1 / 2}, \chi\right\rangle_{H^{\sigma}}-\left\langle L \widehat{w}_{h}^{n}-L w_{h}^{n-1 / 2}, \chi\right\rangle_{H^{\sigma}} \\
& =\left\langle F\left(\bar{U}^{n}\right), \chi\right\rangle_{H^{\sigma}}-\left\langle u_{t}^{n-1 / 2}, \chi\right\rangle_{H^{\sigma}}-\left\langle\bar{\partial} w_{h}^{n}-u_{t}^{n-1 / 2}, \chi\right\rangle_{H^{\sigma}} \\
& -\left\langle L u^{n-1 / 2}, \chi\right\rangle_{H^{\sigma}}-\left\langle L \widehat{u}^{n}-L u^{n-1 / 2}, \chi\right\rangle_{H^{\sigma}} \\
& =\left\langle F\left(\bar{U}^{n}\right)-F\left(u^{n-1 / 2}\right), \chi\right\rangle_{H^{\sigma}}-\left\langle\bar{\partial} w_{h}^{n}-u_{t}^{n-1 / 2}, \chi\right\rangle_{H^{\sigma}} \\
& -\left\langle L \widehat{u}^{n}-L u^{n-1 / 2}, \chi\right\rangle_{H^{\sigma}},
\end{aligned}
$$

using the reproducing kernel property $\left\langle L u^{n}, \chi\right\rangle_{H^{\sigma}}=\left\langle L w_{h}^{n}, \chi\right\rangle_{H^{\sigma}}, \chi \in V_{X}$. Setting $\chi=\widehat{\theta}^{n}$ and using that $\left\langle L \widehat{\theta}^{n}, \widehat{\theta}^{n}\right\rangle_{H^{\sigma}} \geq 0$, leads to

$$
\left\langle\bar{\partial} \theta^{n}, \widehat{\theta}^{n}\right\rangle_{H^{\sigma}}=\frac{1}{2} \bar{\partial}\left\|\theta^{n}\right\|_{H^{\sigma}}^{2}=\bar{\partial}\left\|\theta^{n}\right\|_{H^{\sigma}} \cdot \widehat{\left\|\theta^{n}\right\|_{H^{\sigma}}} \leq R_{n}\left\|\widehat{\theta}^{n}\right\|_{H^{\sigma}} \leq R_{n} \widehat{\left\|\theta^{n}\right\|_{H^{\sigma}}}
$$


with

$$
R_{n}=C_{L}\left\|\bar{U}^{n}-u^{n-1 / 2}\right\|_{H^{\sigma}}+\left\|\bar{\partial} w_{h}^{n}-u_{t}^{n-1 / 2}\right\|_{H^{\sigma}}+\left\|L \widehat{u}^{n}-L u^{n-1 / 2}\right\|_{H^{\sigma}} .
$$

Hence, we have

$$
\bar{\partial}\left\|\theta^{n}\right\|_{H^{\sigma}} \leq R_{n} .
$$

We are now going to bound the three terms defining $R_{n}$ separately. For the first term, we use the fact that $\bar{u}^{n}$ is the value at $t=t_{n-1 / 2}$ of the linear polynomial that interpolates $\left(t_{n-2}, u_{n-2}\right)$ and $\left(t_{n-1}, u_{n-1}\right)$. Hence, we can use the standard error formula for linear interpolation to derive

$$
\begin{aligned}
\left\|\bar{U}^{n}-u^{n-1 / 2}\right\|_{H^{\sigma}} \leq & \left\|\bar{\theta}^{n}\right\|_{H^{\sigma}}+\left\|\bar{\rho}^{n}\right\|_{H^{\sigma}}+\left\|\bar{u}^{n}-u^{n-1 / 2}\right\|_{H^{\sigma}} \\
\leq & \left\|\bar{\theta}^{n}\right\|_{H^{\sigma}}+c h^{\sigma}\left(\left\|u^{n-1}\right\|_{H^{2 \sigma}}+\left\|u^{n-2}\right\|_{H^{2 \sigma}}\right) \\
& +c \tau^{2} u_{t t}\left(\widetilde{t}_{n}\right),
\end{aligned}
$$

where $\widetilde{t}_{n} \in[0, T]$. For the second term, we note that

$$
\begin{aligned}
\left\|\bar{\partial} w_{h}^{n}-u_{t}^{n-1 / 2}\right\|_{H^{\sigma}} & \leq\left\|\bar{\partial} \rho^{n}\right\|_{H^{\sigma}}+\left\|\bar{\partial} u^{n}-u_{t}^{n-1 / 2}\right\|_{H^{\sigma}} \\
& \leq C h^{\sigma}\left\|u_{t}\left(t_{n}^{\prime}\right)\right\|_{H^{2 \sigma}}+C \tau^{2}\left\{\left\|u_{t t t}\left(t_{n}^{\prime \prime}\right)\right\|_{H^{\sigma}}+\left\|u_{t t t}\left(t_{n-1}^{\prime \prime}\right)\right\|_{H^{\sigma}}\right\},
\end{aligned}
$$

where we have used $\bar{\partial} \rho^{n}=\left(\rho^{n}-\rho^{n-1}\right) / \tau=\rho_{t}\left(t_{n}^{\prime}\right)$ with a $t_{n}^{\prime} \in\left[t_{n-1}, t_{n}\right]$, Theorem 4.3 and once again the standard error formula for linear interpolation with $t_{n}^{\prime \prime}, t_{n-1}^{\prime \prime} \in$ $[0, T]$.

The standard difference approximation shows that there is a ${\widetilde{t_{n}}}^{\prime} \in[0, T]$ such that the third term can be bounded by

$$
\left\|L \widehat{u}^{n}-L u^{n-1 / 2}\right\|_{H^{\sigma}} \leq \tau^{2}\left\|L u_{t t}\left({\widetilde{t_{n}}}^{\prime}\right)\right\|_{H^{\sigma}} .
$$

Hence, taking this all into account, we have

$$
\bar{\partial}\left\|\theta^{n}\right\|_{H^{\sigma}} \leq C\left\|\bar{\theta}^{n}\right\|_{H^{\sigma}}+C(u)\left(\tau^{2}+h^{\sigma}\right),
$$

which means

$$
\left\|\theta^{n}\right\|_{H^{\sigma}} \leq(1+C \tau)\left\|\theta^{n-1}\right\|_{H^{\sigma}}+C \tau\left\|\theta^{n-2}\right\|_{H^{\sigma}}+C(u) \tau\left(\tau^{2}+h^{\sigma}\right),
$$

from which we can conclude that

$\left\|\theta^{n}\right\|_{H^{\sigma}}+C \tau\left\|\theta^{n-1}\right\|_{H^{\sigma}} \leq(1+2 C \tau)\left[\left\|\theta^{n-1}\right\|_{H^{\sigma}}+C \tau\left\|\theta^{n-2}\right\|_{H^{\sigma}}\right]+C(u) \tau\left(h^{\sigma}+\tau^{2}\right)$,

which resolves to

$$
\begin{aligned}
\left\|\theta^{n}\right\|_{H^{\sigma}} & \leq\left\|\theta^{n}\right\|_{H^{\sigma}}+C \tau\left\|\theta^{n-1}\right\|_{H^{\sigma}} \\
& \leq(1+2 C \tau)^{n}\left[\left\|\theta^{1}\right\|_{H^{\sigma}}+C \tau\left\|\theta^{0}\right\|_{H^{\sigma}}\right]+C(u) \tau\left(h^{\sigma}+\tau^{2}\right) \sum_{j=0}^{n-1}(1+2 C \tau)^{j} \\
& =(1+2 C \tau)^{n}\left[\left\|\theta^{1}\right\|_{H^{\sigma}}+C \tau\left\|\theta^{0}\right\|_{H^{\sigma}}\right]+C(u) \tau\left(h^{\sigma}+\tau^{2}\right) \frac{(1+2 C \tau)^{n}-1}{2 C \tau} .
\end{aligned}
$$

For a fixed time interval $[0, T]$ this leads, because of $\tau=T / N_{T}$, to

$$
\left\|\theta^{n}\right\|_{H^{\sigma}} \leq C(T)\left[\left\|\theta^{1}\right\|_{H^{\sigma}}+\left\|\theta^{0}\right\|_{H^{\sigma}}+C(u)\left(h^{\sigma}+\tau^{2}\right)\right] .
$$

Next, we bound $\left\|\theta^{1}\right\|_{H^{\sigma}}$. To this end, we have $\theta^{1}=U^{1}-w_{h}^{1}$ and $\theta^{1,0}=U^{1,0}-w_{h}^{1}$ and set $\theta^{0,0}=\theta^{0}=U^{0}-w_{h}^{0}$ and let the operators act as in $\bar{\partial} \theta^{1,0}=\left(\theta^{1,0}-\theta^{0,0}\right) / \tau$ and so on. 
The previous part of the proof applied to this situation, taking (5.6) into account yields the estimate

$$
\bar{\partial}\left\|\theta^{1,0}\right\|_{H^{\sigma}} \leq C\left\|U^{0}-u^{1 / 2}\right\|_{H^{\sigma}}+C(u)\left(h^{\sigma}+\tau^{2}\right) .
$$

Moreover, we have

$$
\left\|U^{0}-u^{1 / 2}\right\|_{H^{\sigma}} \leq\left\|\theta^{0}\right\|_{H^{\sigma}}+\left\|\rho^{0}\right\|_{H^{\sigma}}+\left\|u^{0}-u^{1 / 2}\right\|_{H^{\sigma}} \leq\left\|\theta^{0}\right\|_{H^{\sigma}}+C(u)\left(h^{\sigma}+\tau\right)
$$

such that we can conclude that

$$
\left\|\theta^{1,0}\right\|_{H^{\sigma}} \leq(1+C \tau)\left\|\theta^{0}\right\|_{H^{\sigma}}+C(u) \tau\left(h^{\sigma}+\tau\right) \leq C\left\|\theta^{0}\right\|_{H^{\sigma}}+C(u)\left(h^{\sigma}+\tau^{2}\right) .
$$

In the same way, we can conclude from (5.7) that

$$
\bar{\partial}\left\|\theta^{1}\right\|_{H^{\sigma}} \leq C\left\|\frac{U^{1,0}+U^{0}}{2}-u^{1 / 2}\right\|_{H^{\sigma}}+C(u)\left(h^{\sigma}+\tau^{2}\right) .
$$

Since, by particularly using (5.9),

$$
\begin{aligned}
\left\|\frac{U^{1,0}+U^{0}}{2}-u^{1 / 2}\right\|_{H^{\sigma}} & \leq\left\|\frac{1}{2}\left(\theta^{1,0}+\theta^{0}\right)\right\|_{H^{\sigma}}+\left\|\widehat{w}_{h}^{1}-u^{1 / 2}\right\|_{H^{\sigma}} \\
& \leq\left\|\frac{1}{2}\left(\theta^{1,0}+\theta^{0}\right)\right\|_{H^{\sigma}}+\left\|\widehat{w}_{h}^{1}-\widehat{u}^{1}\right\|_{H^{\sigma}}+\left\|\widehat{u}^{1}-u^{1 / 2}\right\|_{H^{\sigma}} \\
& \leq C\left\|\theta^{0}\right\|_{H^{\sigma}}+C(u)\left(h^{\sigma}+\tau^{2}\right),
\end{aligned}
$$

we can conclude from (5.10) that

$$
\left\|\theta^{1}\right\|_{H^{\sigma}} \leq(1+C \tau)\left\|\theta^{0}\right\|_{H^{\sigma}}+C(u) \tau\left(h^{\sigma}+\tau^{2}\right)
$$

and the bound $\left\|\theta^{0}\right\|_{H^{\sigma}} \leq C(u) h^{\sigma}$ together with (5.8) establishes the result.

\section{Conclusion}

In this paper, we considered reaction diffusion systems and their numerical approximation. For discretisation in space we used spherical basis functions, which are reproducing kernels in smooth Sobolev spaces. The most prominent example of such SBFs are given by the restriction of Wendland's functions to the sphere. We identified the norm-minimal collocation as a Galerkin method in a specific Sobolev space. We proved convergence and stability for the semi-discrete approximation. The convergence order proven is induced by the smoothness of the involved functions and in this sense optimal. Finally, we proved convergence for the fully discrete system if a linearised Crank-Nicolson method is used in time. This, however, is only an example to show how standard techniques have to be modified to prove convergence for higher order time discretisations.

\section{REFERENCES}

1. S. M. Allen and J. W. Cahn, A macroscopic theory for antiphase boundary motion and its application to antiphase domain coarsing, Acta. Metal. 27 (1979), 1085-1095.

2. T. Belytschko, Y. Krongauz, D. Organ, M. Fleming, and P. Krysl, Meshless methods: An overview and recent developments, Comput. Methods in Appl. Mechanics and Engineering 139 (1996), 3-47.

3. H. Brezis and P. Mrionescu, Composition in fractional Sobolev spaces, Discrete and Continuous Dynamical Systems 7 (2001), 241-246. MR1808397 (2002c:46063)

4. G. Fasshauer, Meshfree approximation methods with MATLAB, World Scientific Publishers, Singapore, 2007. MR2357267 (2008i:65002)

5. N. Flyer and B. Fornberg, Radial basis functions: Developments and applications to planetary scale flows, Computers and Fluids 46 (2011), 23-32. 
6. N. Flyer and G. Wright, Transport schemes on a sphere using radial basis functions, J. Comp. Phys. 226 (2007), 1059-1084. MR2356868 (2008h:86011)

7. __ A radial basis function method for the shallow water equations on a sphere, Proc. R. Soc. A 465 (2009), 1949-1976. MR2500804 (2010g:76093)

8. B. Fornberg and E. Lehto, Stabilization of RBF-generated finite difference methods for convective PDEs, J. Comput. Phys. 230 (2011), 2270-2285. MR2764546

9. Q. T. Le Gia, Approximation of parabolic PDEs on spheres using collocation method, Adv. Comput. Math. 22 (2005), 377-397. MR2131154(2006a:65130)

10. Q. T. Le Gia, F. J. Narcowich, J. D. Ward, and H. Wendland, Continuous and discrete leastsquares approximation by radial basis functions on spheres, J. Approx. Theory 143 (2006), 124-133. MR2271729(2007k:41078)

11. T. M. Morton and M. Neamtu, Error bounds for solving pseudodifferential equatons on spheres by collocation with zonal kernels, J. Approx. Theory 114 (2002), 242-268. MR.1883408 (2002k:65200)

12. C. Müller, Spherical Harmonics, Springer, Berlin, 1966. MR0199449 (33:7593)

13. F. J. Narcowich, X. Sun, J. D. Ward, and H. Wendland, Direct and inverse Sobolev error estimates for scattered data interpolation via spherical basis functions, J. Foundations of Computational Mathematics 7 (2007), 369-390. MR2335250 (2008i:41023)

14. F. J. Narcowich and J. D. Ward, Scattered data interpolation on spheres: Error estimates and locally supported basis functions, SIAM J. Math. Anal. 33 (2002), 1393-1410. MR1920637 (2003j:41021)

15. J. E. Pearson, Complex patterns in a simple system, Science 261 (1993), 189-192.

16. M. E. Taylor, Partial differential equations. III, Applied Mathematical Sciences, vol. 117, Springer, New York, 1997. MR 1477408 (98k:35001)

17. V. Thomée, Galerkin finite element methods for parabolic problems, second ed., Springer, Berlin, 2006. MR2249024 (2007b:65003)

18. V. Thomée and L. Wahlbin, On Galerkin methods in semilinear parabolic problems, SIAM J. Numer. Anal. 12 (1975), 378-389. MR0395269 (52:16066)

19. H. Wendland, Scattered data approximation, Cambridge Monographs on Applied and Computational Mathematics, Cambridge University Press, Cambridge, UK, 2005. MR.2131724 (2006i:41002)

Mathematical Institute, University of Oxford, 24-29 St Giles', Oxford, OX1 3LB, UNITED KINGDOM

E-mail address: holger.wendland@maths.ox.ac.uk 